\title{
Weakly imbalanced strong turbulence
}

\section{Andrey Beresnyak}

\author{
Los Alamos National Laboratory, Los Alamos, NM 87544
}

\begin{abstract}
The theory of strong MHD turbulence with cross-helicity has been a subject of many recent studies. In this paper we focused our attention on low-imbalance limit and performed high-resolution 3D simulations. The results suggest that in this limit both $\mathbf{w}^{+}=\mathbf{v}+\mathbf{b}$ and $\mathbf{w}^{-}=\mathbf{v}-\mathbf{b}$ are cascaded strongly. The model for imbalance based on so-called "dynamic alignment" strongly contradicts numerical evidence.
\end{abstract}

Keywords. MHD - turbulence - ISM: kinematics and dynamics

\section{Introduction}

MHD turbulence has attracted attention of astronomers since mid 1960s. As most astrophysical media are ionized, plasmas are coupled to the magnetic fields (Biskamp, 2003). One-fluid MHD is applicable to most astrophysical environments on macroscopic scales. Turbulence covering huge range of scales has been observed in the ISM (Armstrong et al., 1995).

As with hydrodynamics which has a "standard" phenomenological model of energy cascade (Kolmogorov, 1941), MHD turbulence has one too. This is the Goldreich-Sridhar model henceforth GS95 that uses a concept of critical balance, which maintains that turbulence will stay marginally strong down the cascade. The spectrum of GS95 is supposed to follow a $-5 / 3$ Kolmogorov scaling. However, a shallower slopes has been reported in numerical studies see, e.g., Müller 2005, which motivated modifications of GS95 (see, e.g., Boldyrev 2005, 2006 and Gogoberidze, 2007).

The other problem of GS95 is that it is incomplete, as it does not treat the most general imbalanced, or cross-helical case. As turbulence is a stochastic phenomenon, an average zero cross helicity does not preclude a fluctuations of this quantity in the turbulent volume. Also, most of astrophysical turbulence is naturally imbalanced, due to the fact that it is generated by a strong localized source of perturbations, such as the Sun in case of solar wind.

Several models of imbalanced turbulence appeared recently: Lithwick et al., 2007 henceforth LGS07, Beresnyak \& Lazarian (2008), Chandran (2008), Perez \& Boldyrev (2009) henceforth PB09. The full self-consistent analytical model for strong turbulence, however, does not yet exist. In this situation observations and direct numerical simulations (DNS) of MHD turbulence will provide necessary feedback to theorists. We concentrated on two issues, namely that a) the power spectrum slopes of MHD turbulence can not be measured directly from available numerical simulations, supporting an earlier claim in BL09b, b) the ratio of Elsässer dissipation rates is a very robust quantity that can differentiate among many imbalanced models.

\section{Numerical setup}

We solved incompressible MHD or Navier-Stokes equations:

$$
\partial_{t} \mathbf{w}^{ \pm}+\hat{S}\left(\mathbf{w}^{\mp} \cdot \nabla\right) \mathbf{w}^{ \pm}=-\nu_{n}\left(-\nabla^{2}\right)^{n / 2} \mathbf{w}^{ \pm}+\mathbf{f}^{ \pm},
$$

where $\hat{S}$ is a solenoidal projection and $\mathbf{w}^{ \pm}$(Elsasser variables) are $\mathbf{w}^{+}=\mathbf{v}+\mathbf{b}$ and $\mathbf{w}^{-}=\mathbf{v}-\mathbf{b}$ where we use velocity $\mathbf{v}$ and magnetic field in velocity units $\mathbf{b}=\mathbf{B} /(4 \pi \rho)^{1 / 2}$. The magnetic Prandtl number here is unity. The RHS of this equation includes a linear dissipation term which is called viscosity or diffusivity for $n=2$ and hyper-viscosity or hyper-diffusivity for $n>2$ 
Table 1. Three-dimensional simulations of balanced and imbalanced turbulence

\begin{tabular}{cccccc}
\hline Run & Resolution: $n_{x} \times n_{y} \times n_{z}$ & Forcing & Dissipation & $\epsilon^{+} / \epsilon^{-}$ & $\left(w^{+}\right)^{2} /\left(w^{-}\right)^{2}$ \\
\hline B1 & $1024 \times 3072^{2}$ & $w^{ \pm}$ & $-3.3 \cdot 10^{-17} k^{6}$ & $\sim 1$ & $\sim 1$ \\
B2 & $768 \times 2048^{2}$ & $v$ & $-3.1 \cdot 10^{-16} k^{6}$ & $\sim 1$ & $\sim 1$ \\
B3 & $768 \times 2048^{2}$ & $w^{ \pm}$ & $-3.1 \cdot 10^{-16} k^{6}$ & $\sim 1$ & $\sim 1$ \\
B4 & $768 \times 2048^{2}$ & $w^{ \pm}$ & $-6.7 \cdot 10^{-5} k^{6}$ & $\sim 1$ & $\sim 1$ \\
I1 & $512 \times 1024^{2}$ & $w^{ \pm}$ & $-1.9 \cdot 10^{-4} k^{2}$ & 1.187 & $1.35 \pm 0.04$ \\
I2 & $768^{3}$ & $w^{ \pm}$ & $-6.8 \cdot 10^{-14} k^{6}$ & 1.187 & $1.42 \pm 0.04$ \\
I3 & $512 \times 1024^{2}$ & $w^{ \pm}$ & $-1.9 \cdot 10^{-4} k^{2}$ & 1.412 & $1.88 \pm 0.04$ \\
I4 & $768^{3}$ & $w^{ \pm}$ & $-6.8 \cdot 10^{-14} k^{6}$ & 1.412 & $1.98 \pm 0.03$ \\
I5 & $1024 \times 1536^{2}$ & $w^{ \pm}$ & $-1.5 \cdot 10^{-15} k^{6}$ & 2 & $5.57 \pm 0.08$ \\
I6 & $1024 \times 1536^{2}$ & $w^{ \pm}$ & $-1.5 \cdot 10^{-15} k^{6}$ & 4.5 & $45.2 \pm 1.5$ \\
\hline
\end{tabular}

and the driving force $\mathbf{f}^{ \pm}$. The total dissipation per unit time of each Elsässer energy $\left(w^{ \pm}\right)^{2}$ by the linear dissipation term are called Elsässer dissipation rates $\epsilon^{ \pm}$. In most simulations from Table 1 we drove $w^{+}$and $w^{-}$randomly and independently. Each $\mathbf{w}$ field can be represented as a mean field $\mathbf{v}_{\mathbf{A}}$ plus perturbation $\mathbf{w}^{ \pm}= \pm \mathbf{v}_{\mathbf{A}}+\delta \mathbf{w}^{ \pm}$. We will also use characteristic perturbation magnitudes on a particular scale $l, w_{l}^{ \pm}$. We solved these equations with a pseudospectral code that was described in great detail in our earlier publications BL09a, BL09b. Table 1 enumerates latest high-resolution runs, which were performed in so-called reduced MHD approximation, where the $\mathbf{w}^{ \pm}$component parallel to the mean field (pseudo-Alfvén mode) is omitted and so are the parallel gradients in the nonlinear term $\left(\left(\delta \mathbf{w}^{\mp} \cdot \nabla_{\|}\right) \delta \mathbf{w}^{ \pm}\right.$. The linear propagation term, i.e. $\left(\left(\delta \mathbf{v}_{\mathbf{A}} \cdot \nabla_{\|}\right) \delta \mathbf{w}^{ \pm}\right.$, however, is always finite. This is because we consider turbulence injected at such $k_{\|}$that $v_{A} k_{\|}$stays finite, in other words, we use computational box which is elongated in $\mathrm{x}$ direction by a factor which is proportional to $v_{A}$. In this situation the actual value of the mean field $B_{0}$ or $v_{A}$ drops out of calculations. Physically this means that $B_{0}$ is "large enough" compared to perturbations. Under these assumptions one studies purely Alfvénic dynamics in a strong mean field, i.e., Alfvénic turbulence.

We started our high resolution simulations with earlier lower-resolution runs that were evolved for a long time, typically hundreds Alfvenic times and reached stationary state. The balanced runs were evolved, typically, for 6-10 Alfvenic times and the imbalanced runs were evolved for longer times, typically 10-40. The energy injection rates were kept constant in I1-6 and the fluctuating dissipation rate was within few percent of the former. The fluctuations in total energies $\left(w^{+}\right)^{2}$ and $\left(w^{-}\right)^{2}$ was a main source of uncertainty for I1-6 shown on Fig. 1 For more simulations and the analysis of anisotropy, see also BL09a, BL09b.

\section{Nonlinear cascading and dissipation rate}

One of the most robust quantities in numerical simulations of MHD turbulence is the energy cascading rate or dissipation rate. In high-Reynolds number turbulence energy has to cascade through many steps before dissipating and the dissipation is negligible on the outer (large) scale. Therefore, the nonlinear energy cascading rate and the dissipation rate are used interchangeably.

In hydrodynamic turbulence the dissipation rate and the spectrum of velocity are connected by the well-known Kolmogorov constant:

$$
E(k)=C_{K} \epsilon^{2 / 3} k^{-5 / 3} .
$$

The important fact that strong hydrodynamic turbulence dissipates in one dynamic timescale $l / v$ is reflected by $C_{K}$ being close to unity $(\sim 1.6)$. In MHD turbulence, however, there are two energy cascades (or "Elsasser cascades") and there are two dissipation rates, $\epsilon^{+}$and $\epsilon^{-}$. The question of how these rates are related to velocity-like Elsasser amplitudes $\mathbf{w}^{+}$and $\mathbf{w}^{-}$is one of the central questions of imbalanced MHD turbulence. Each model of strong imbalanced 


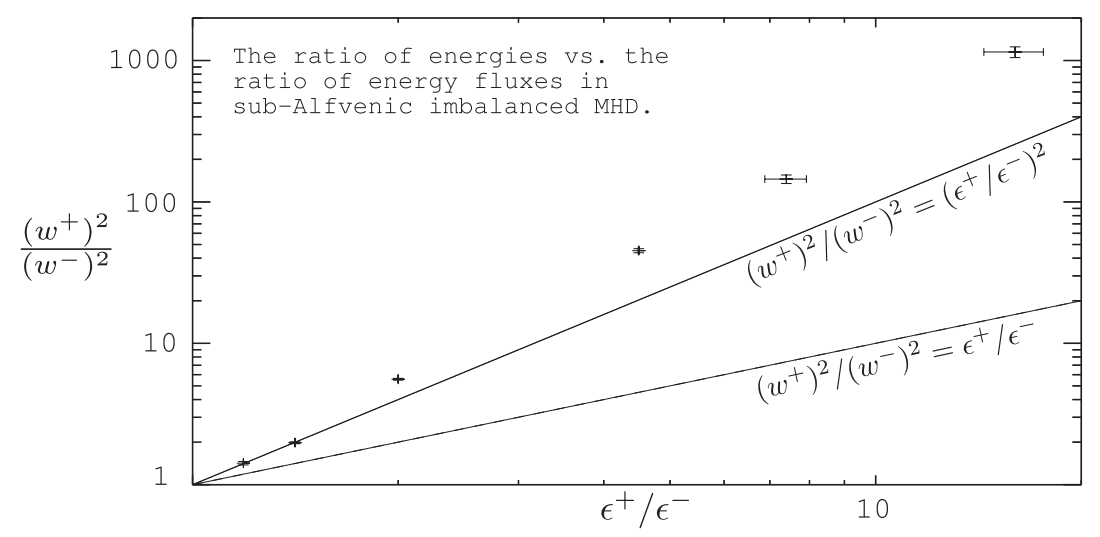

Figure 1. Energy imbalances versus dissipation rate imbalance. Predictions, solid: LGS07, dashed: PB09. Errorbars indicate fluctuation in time. On this plot I1 and I3 are omitted as they are close to I2 and I4. I1 and I3 are simulations with normal viscosity which have slightly lower energy imbalance than I2 and I4, see Table 1. This is an indication that in these simulations viscosity was affecting outer scales. Two high imbalance points are taken from BL09a. For a fixed dissipation ratio the energy imbalance has a tendency to only increase with resolution.

turbulence advocates a different physical picture of cascading and provides a different relation between the ratio of energies $\left(w^{+}\right)^{2} /\left(w^{-}\right)^{2}$ and the ratio of fluxes $\epsilon^{+} / \epsilon^{-}$.

Goldreich-Sridhar model (GS95) predicts that in the balanced case the cascading is strong and each wave is cascaded by the shear rate of the opposite wave, i.e.,

$$
\epsilon^{+}=\frac{\left(w_{l}^{+}\right)^{2} w_{l}^{-}}{l}, \quad \epsilon^{-}=\frac{\left(w_{l}^{-}\right)^{2} w_{l}^{+}}{l} .
$$

It is similar to Kolmogorov cascade with w's replacing $v$. Although this model does not make predictions for the imbalanced case, one could hope that in the case of small imbalance these formulae will still work. In this case we will obtain $\left(w^{+}\right)^{2} /\left(w^{-}\right)^{2}=\left(\epsilon^{+} / \epsilon^{-}\right)^{2}$. LGS07 argued that this relation will hold even for large imbalances.

For the purpose of this short paper we mostly discuss the prediction of LGS07, $\left(w^{+}\right)^{2} /\left(w^{-}\right)^{2}=$ $\left(\epsilon^{+} / \epsilon^{-}\right)^{2}$ and the prediction of PB09 that nonlinear timescales are equal for both waves, which effectively lead to $\dagger\left(w^{+}\right)^{2} /\left(w^{-}\right)^{2}=\epsilon^{+} / \epsilon^{-}$. Note, that the last prediction is also true for highly viscous flows $\left(R e=R e_{m} \ll 1\right)$. It could be rephrased that PB09 predicts turbulent viscosity which is equal for both components.

Compared to spectral slopes, dissipation rates are robust quantities that require much smaller dynamical range and resolution to converge. Fig. 1 shows energy imbalance $\left(w^{+}\right)^{2} /\left(w^{-}\right)^{2}$ versus dissipation rate imbalance $\epsilon^{+} / \epsilon^{-}$for simulations I2, I4, I5 and I6. We also use two data points from our earlier simulations with large imbalances, A7 and A5 from BL09a. I1 and I3 are simulations with normal viscosity similar to I2 and I4. They show slightly smaller energy imbalances than I2 and I4 (Table 1).

We see that most data points are above the line which is the prediction of LGS07. In other words, one can deduce that numerics strongly suggest that

$$
\frac{\left(w^{+}\right)^{2}}{\left(w^{-}\right)^{2}} \geqslant\left(\frac{\epsilon^{+}}{\epsilon^{-}}\right)^{2} .
$$

Although there is a tentative correspondence between LGS07 and the data for small degrees of imbalance, the deviations for large imbalances are significant. In the case of strong imbalances

$\dagger$ Both of these predictions are subject to intermittency corrections. We average $\left(w^{+}\right)^{2}$ and $\left(w^{-}\right)^{2}$ over volume and time. This averaging does not take into account possible fluctuations in $\epsilon^{+}$and $\epsilon^{-}$. We believe, however, that these effects are small, as long as we use the second-order measures, such as energy. 


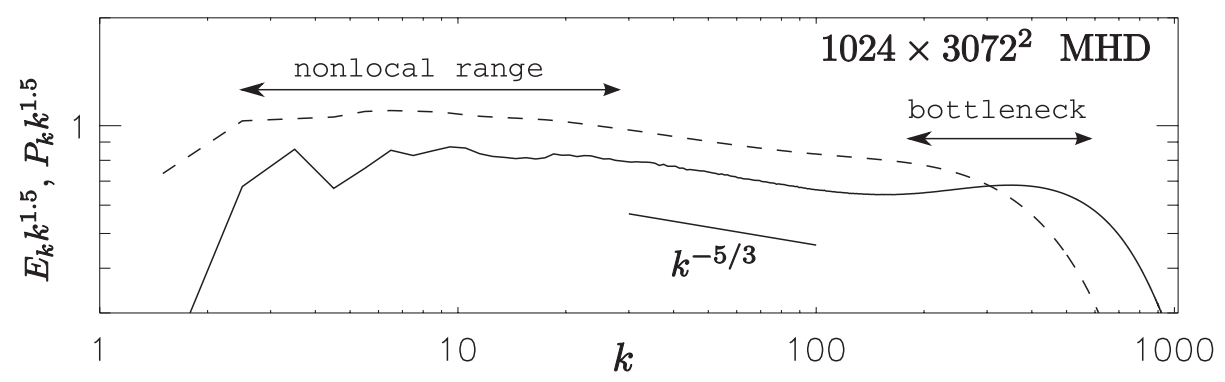

Figure 2. The spectrum from B1. Due to a higher resolution we are starting to see an asymptotic $-5 / 3$ slope of MHD turbulence. The Kolmogorov constant for this purely Alfvenic turbulence is $C_{K} \approx 3.2$, which is much higher than for hydro turbulence. This fact was missed in earlier lower resolution simulations (Biskamp, 2003). Also, higher $C_{K}$ means less efficient energy transfer that is fully consistent with our picture of diffuse locality (BL10).

data deviates from GS95 and LGS07 and suggests that the strong component cascading rate is smaller than what is expected from strong cascade (BL08).

As to PB09 prediction, it is inconsistent with data for all degrees of imbalance including those with small imbalance and normal viscosity, i.e. I1 and I3.

\section{Discussion}

One of the most important measures not mentioned in this paper is the anisotropy of MHD turbulence. It had been considered in great detail in our earlier publication BL09a. In particular, we refer the reader to the result of BL08, BL09a that the anisotropy of strong component is smaller than the anisotropy of weak component. This fact is inconsistent with both the naive application of GS95 critical balance (which would have predicted the opposite), or the derivation in LGS07 that suggests that the both waves have the same anisotropy.

PB09 claims that the nonlinear timescales for both components are equal, i.e. there is a turbulent viscosity which is the same for both components, regardless of the degree of imbalance. This seems counter-intuitive for transition to freely-propagating Alfvenic waves. PB09 formula, $w^{+} / w^{-}=\sqrt{\epsilon^{+} / \epsilon^{-}}$suggests that the asymptotic $\left(R e=R e_{m} \gg 1\right)$ prediction for energy imbalance in this case will be the same as in highly viscous case $\left(R e=R e_{m} \ll 1\right)$, i.e. $\left(w^{+}\right)^{2} /\left(w^{-}\right)^{2}=$ $\epsilon^{+} / \epsilon^{-}$. This is at odds with numerical evidence, which suggests $\left(w^{+}\right)^{2} /\left(w^{-}\right)^{2} \geqslant\left(\epsilon^{+} / \epsilon^{-}\right)^{2}$.

\section{References}

Aluie, H. \& Eyink, G. L., 2010, Phys. Rev. Lett. 104, 081101

Armstrong, J. W., Rickett, B. J. \& Spangler, S. R. 1995, ApJ, 443, 209

Beresnyak, A. \& Lazarian, A. 2008, ApJ, 678, 961 (BL08)

Beresnyak, A. \& Lazarian, A. 2009a, ApJ, 702, 460 (BL09a)

Beresnyak, A. \& Lazarian, A. 2009b, ApJ, 702, 1190 (BL09b)

Beresnyak, A. \& Lazarian, A. 2010, ApJ, 722, L110 (BL10)

Biskamp, D. 2003, Magnetohydrodynamic Turbulence. (Cambridge: CUP)

Boldyrev, S. 2005, ApJ, 626, L37; Boldyrev, S. 2006, Phys. Rev. Lett., 96, 115002

Chandran, B. D. G. 2008, ApJ, 685, 646

Gogoberidze, G. 2007, Phys. Plasmas, 14, 022304

Goldreich, P. \& Sridhar, S. 1995, ApJ, 438, 763

Kolmogorov, A. 1941, Dokl. Akad. Nauk SSSR, 31, 538

Lithwick, Y., Goldreich, P., \& Sridhar, S. 2007, ApJ, 655, 269 (LGS07)

Müller W.-C. \& Grappin, R 2005, Phys. Rev. Lett., 95, 114502

Perez, J. \& Boldyrev, S. 2009, Phys. Rev. Lett., 102, 025003, (PB09) 